\title{
Investigation on low cycle fatigue behaviours of Mg-12Gd-3Y-0.5Zr magnesium alloy
}

\author{
Xiao Ming $\mathrm{YANG}^{1, a}$ \\ ${ }^{1}$ Jingchu University of Technology, JinMen City, HuBei province, china
}

\begin{abstract}
In this paper, low cycle fatigue behaviours of $\mathrm{Mg}-12 \mathrm{Gd}-3 \mathrm{Y}-0.5 \mathrm{Zr}$ magnesium alloy under different strain amplitudes were investigated. It shows that Mg-12Gd-3Y-0.5Zr magnesium alloy has good cyclic stabilization. SEM fracture micrograph indicate that fatigue cracks initiate on the surface or subsurface. Low cycle fatigue mechanism of Mg-12Gd-3Y-0.5Zr magnesium alloy under different total strain amplitudes is disscussed in this paper.
\end{abstract}

\section{Introduction}

More and more importance is paid to magnesium alloys due to their high specific strength, good machinability and castability [1]. Many measures have been adopted to improve their mechanical properties [1]. However, magnesium alloys are limited to some noncritical parts as a result of the restriction of fatigue strength and creep resistance at high temperatures [2]. With addition of rare earth elements, Mg-12Gd-3Y- $0.5 \mathrm{Zr}$ alloy show high tensile strength and modified fatigue strength at elevated temperatures.

In the present day, investigation on low cycle fatigue behaviours of magnesium alloys is only limited to $\mathrm{AZ}$ series and AM series. Begum investigated low cycle fatigue behaviours of as-rolled AZ31 magnesium alloy and the research indicates that it shows cyclic stabilization under low strain amplitudes and cyclic hardening under high strain amplitudes, respectively [3]. Park revealed that low cycle fatigue behaviours of AZ31 magnesium alloy with strong basal plane texture was predominated by the alternation of twinning and detwinning during each cycle [4].

In this paper, the cyclic strain response and fatigue machanism is disscussed.

\section{Experimental}

The ingots of $\mathrm{Mg}-12 \mathrm{Gd}-3 \mathrm{Y}-0.5 \mathrm{Zr}$ alloy(hereinafter referred to as GW123K) were rolled with a ratio of $25: 1$ at $400^{\circ} \mathrm{C}$. The second phase whose constituent is approately $\operatorname{Mg}_{24}(\mathrm{Gd}, \mathrm{Y})_{5}[5]$ was precipitated in microstructre in the process of extrusion.

Specimens for low cyclic fatigue tests were cut by electric spark machining along the rolling direction with a gauge sections of $5 \times 4.5 \times 14 \mathrm{~mm}^{3}$. Low cycle fatigue testss controlled by total strain range from $1.5 \times 10^{-3} \sim$ $2 \times 10^{-2}$ were carried out on an MTS 810 fatigue testing machine with an immanent frequncy of $0.5 \mathrm{~Hz}$.

The fracture surface morphologies of the specimens were observed by scanning electron microscopy (SEM, QUANTA600).

\section{Experimental results and discussion}

\subsection{Microstructure}

Fig.1(a) shows optical micrograph of microstructure for GW123K. It can be seen that microstructure of GW123K is composed of $\alpha-\mathrm{Mg}$ solid solution and $\mathrm{Mg}$-riched precipitation which precipitaed along or inside grain. The average grain size of as-rolled GW123K is about $10 \mu \mathrm{m}$ and many grains are characteristic of recrystallization. It can be seen from Fig.1(b) that there are a large quantity of uniformly distributed second precipitates in microstructure of GW123K alloy.

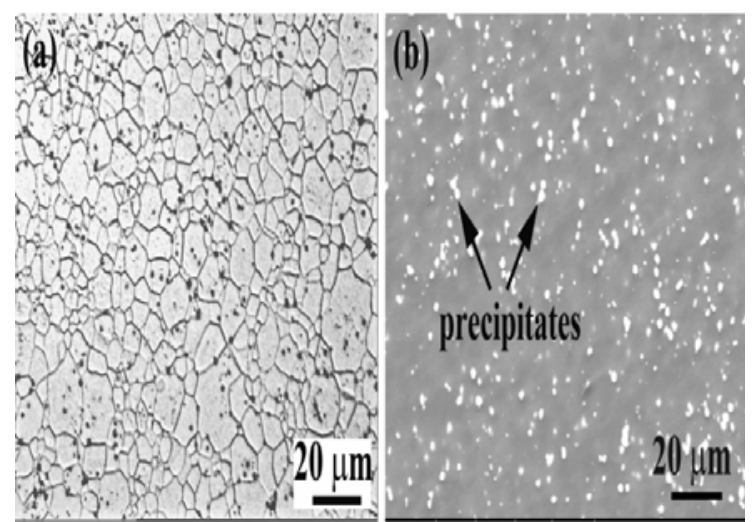

Figure 1. Microstructures of GW123k alloy, (a) OM and (b) SEM backscatter image where abundant fine precipitates can be observed.

\footnotetext{
${ }^{\mathrm{a}}$ Corresponding author: yang760507@163.com. This research was supported by university's scientific research project of JingChu University of Technology(No. 2013BK02)
} 


\subsection{Cyclic stress response curve}

Cyclic stress amplitude vs number of cycles for GW123K at different total strain. Amplitudes are shown in Fig.2. It indicates that GW123K maitain cyclic response stability which is different from cyclic hardening of other rolled magnesium alloys [6] and cyclic sofening of as-ECAPed magnesium alloys [7]. It is well known that both cyclic hardening and cyclic sofening depend on dislocation movement to a great extent. As a result of pinning effect of solid solution and precitation phase, dislocation density of rolled GW123K is not prone to propogate in the pocess of low cycle fatigue teses which impair cyclic hardening. Furthermore, initiation and propogation of fatigue cracks under cyclic loading will lead to loss of loaded area which causes a little cyclic sofening. In general, GW123K alloy shows cyclic response stablity as a combination of interaction of cyclic hardening and cyclic sofening 'mention above.

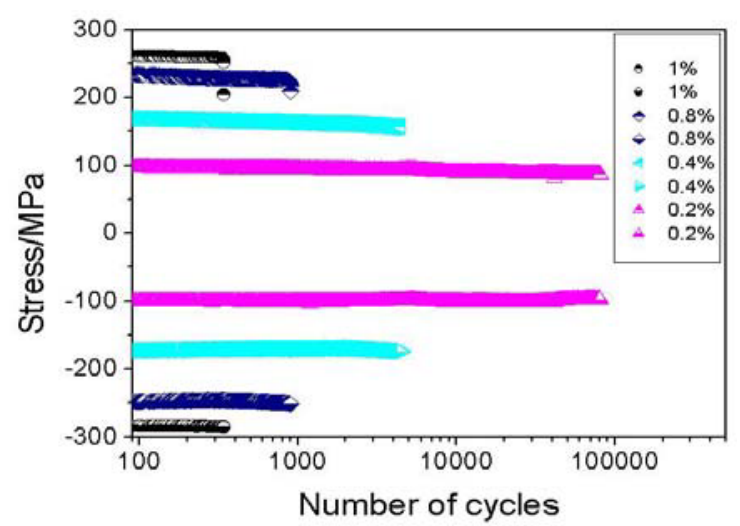

Figure 2. Cyclic stress amplitude vs number of cycles for GW123K at different total strain. amplitudes.

\subsection{Hysteresis loop}

Fig.3 shows hysteresis loops of GW123k alloy at the half-life cycle for different total strain amplitudes. As a result of its cyclic response stability, the shape of hysteresis loop remains unchanged. However, the shape of hysteresis loop is linear when strain amplitude is less than $0.2 \%$ which indicates that its cyclic deformation is mainly composed of plastic deformation.

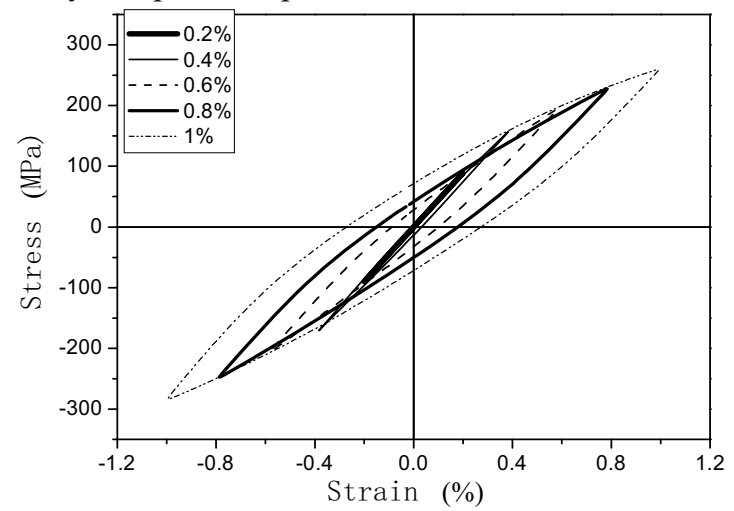

Figure 3. Hysteresis loops of GW123k alloy at the half-life cycle for different total strain amplitudes.

\subsection{Strain-lifetime curves}

Strain amplitude vs number of cycles to failure curves is shown in Fig.4. It can be seen from Fig.4(a) that both elastic strain and plastic strain are linear relationship with reversals to failure and all of them abide by Basquin and Coffin-Manson equations. Fig.4(b) indicates that low cycle fatigue properties of $\mathrm{GW} 123 \mathrm{~K}$ is lower than aluminium alloy but has the same conditon as that of other magnesium alloys. The prediction equation of low cycle fatigue of GW123K is as follows :

$$
\begin{aligned}
\frac{\Delta \varepsilon_{e}}{2} & =0.2076\left(2 N_{f}\right)^{-0.218} \\
\frac{\Delta \varepsilon_{p}}{2} & =1.353\left(2 N_{f}\right)^{-0.956}
\end{aligned}
$$

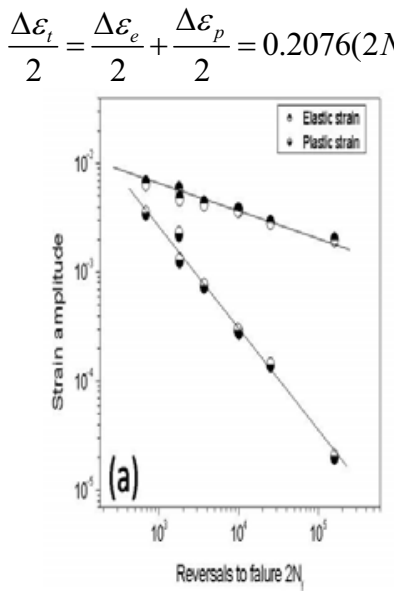

Figure 4. Strain amplitude vs number of cycles to failure curves for (a) as-rolled GW123k, (b) for different magnesium alloys and an aluminium alloy.

\subsection{Analysis of low cycle fatigue mechanism}

SEM images of overall fracture surfaces of specimens are shown in Fig.5. The fracture surface is very smooth and characteristic of fatigue failure. Fatigue cracks initiate from surface or subsurface and they initiate one or more faigue origins (shown as indicated by the arrows).

Fig.6 shows SEM images of fracture surfaces of two specimens and corresponding energy spectrum analysis of fatigue crack initiate sites. It can be seen that fatigue cracks initiate from Gd-riched intermetallic compounds with large size or large-sized inclusions(mainly composed of oxides of $\mathrm{Mg}$ and $\mathrm{Gd}$ ). Stress concentration caused by intermetallic compounds and inclusions induces fatigue initiation which result in final fatigue failure of GW123K alloy under cyclic loading. A conclusion can be drawn that large-sized intermetallic compounds and inclusions impair fatigue property of GW123K alloy. As a result, fatigue cracks initiating from large-sized intermetallic compounds and inclusions is the dominated fatigue mechanism for GW123K under low cycle fatigue tests. 


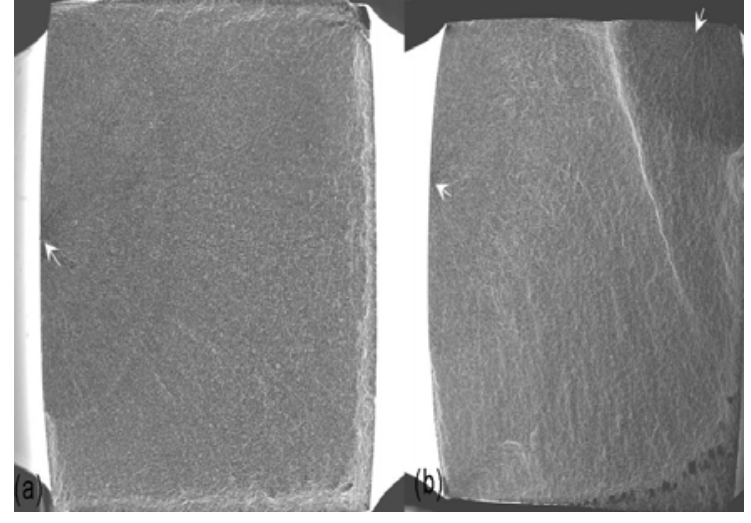

Figure 5. SEM images of overall fracture surfaces of specimens: (a) fatigue crack initiates from single origin, (b) fatigue crack initiates from two origins(fatigue origin shown as indicated by the arrows)

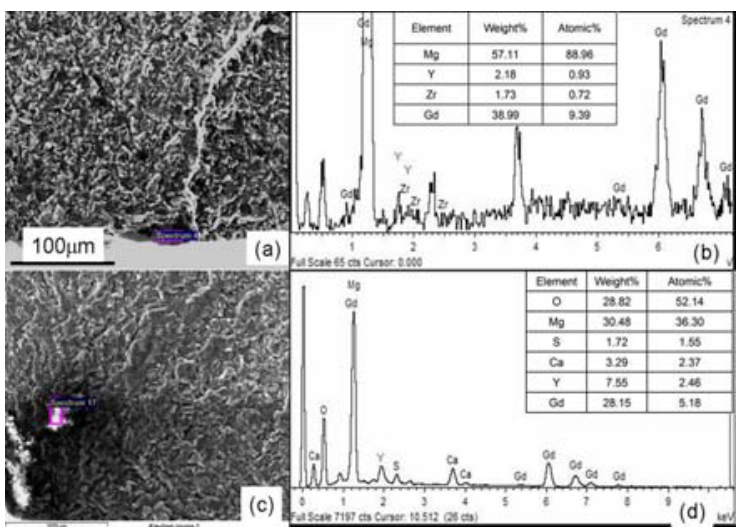

Figure 6. SEM images of fracture surfaces of two specimens and corresponding energy spectrum analysis of fatigue crack initiate sites (a) large second-phase, (b) composition of second-phase in (a), (c)oxide inclusion, (d) composition of oxide inclusion in (c).

Fig .7 shows fatigue fracture surface for specimen fatigued at strain amplitude of $0.2 \%$. It can be seen that fatigue fracture can be divided into three obvious regions, that is to say, region I of fatigue cracks initiation and stable propagation, region II of unstable crack propagation and region III of instaneous failure. region I has typical feature of transcrystalline fracture in which the fracture surface is very complanate and mainly composed of cleavage planes (as shown in Fig.7(b)). A small amount of shallow dimples and cleavage planes can be observed in region II (as shown in Fig.7(c)) and fracture surface in this region is coarse. Region III(as shown in Fig.7(d)) has typical feature of a lot of shallow dimples and some second phase particle can be observed in the bottom of dimples which shows that GW123K has poor plasticity .

Fatigue fracture surface for specimen fatigued at strain amplitude of $0.5 \%$ is shown in Fig. 8 . It can be seen from frature morphology that there is a transitional region between region I and region III. Compared with fatigue fracture under low strain amplitude fagitue tests, volume fraction of clevage planes decreased to a great extent and fracture surface became more coarse than that of the former. There are a bit more dimples in transitional region but no large-sized transcrystalline clevage plane can be observed in this transitional region. The characteristic of region III is similar to that of specimen fatigued at strain amplitude of $0.2 \%$, and the differences between them are that the latter has smaller and fewer dimples.

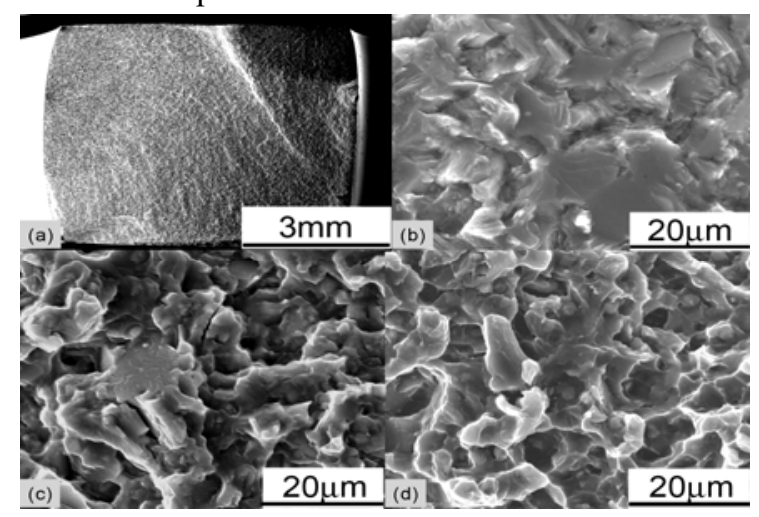

Figure 7. Fatigue fracture surface for specimen fatigued at strain amplitude of $0.2 \%$ : ( a ) overall fracture surface, (b) Region I denotes fatigue crack initiation and steady propagation region; (b) Region II denotes transition region of fatigue crack propagate from steady to unstable; (d) Region III denotes fatigue crack unstable propagation and final failure.

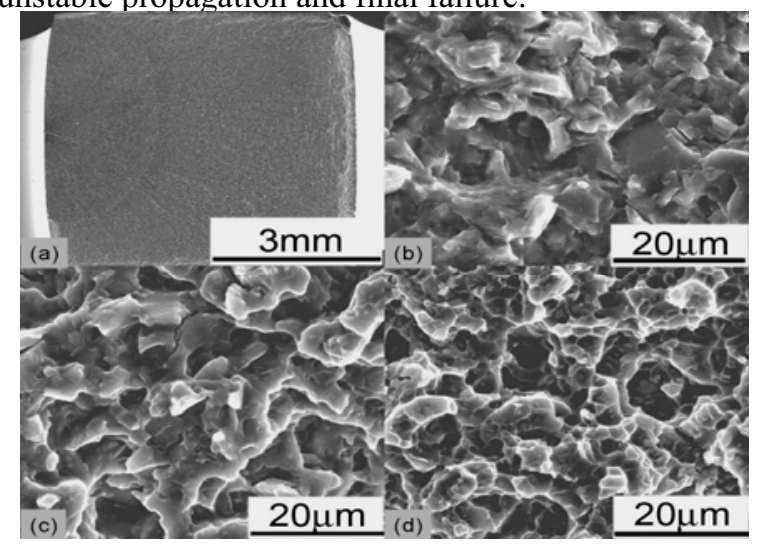

Figure 8. Fatigue fracture surface for specimen fatigued at strain amplitude of $0.5 \%$ : ( a ) overall fracture surface, (b) Region I denotes fatigue crack initiation and steady propagation; (b) Region II denotes transition region of fatigue crack propagate from steady to unstable; (d) Region III denotes fatigue crack unstable propagation and final failure.

\section{Conclusions}

(1) GW123K alloy has charactersitic of cyclic stress response stability in the process of low cycle fatigue tests controlled by strain.

(2) Both elastic strain and plastic strain of GW123K abide by Basquin equation and Coffin-Manson equation, respectively. Low cycle fatigue property of GW123K is close to that of AZ31 and AM50 magnesium alloys. 
(3) Fatigue cracks initiating from large-sized intermetallic compounds and inclusions is the dominated fatigue fracture mechanism.

(4) SEM observations on fatigue fracture surface show that it has three typical fatigue cracks propagation regions, and with the increase of strain amplitude it has smaller and fewer dimples.

\section{Acknowledgement}

This work was funded by scientific research project of Jingchu University of Technology (NO. 2013BK02) and partly by subject groupings of green chemical industry and biological medicine. We would like to thank Yang Fan for kind assistance on SEM and TEM analysis.

\section{References}

1. W. J. Kim, S. W. Chung, C. S. Chung and D. Kum. Acta Mater. 49, 3337 (2001)

2. A.A. Luo, Int.Mater.Rev. 49, 13(2004

3. S. Begum, D. L. Chen, S. Xu, A.A. Luo. Inter. J. Fatigue. 31, 726 (2009)

4. S. H. Park, S. G. Hong, B. H. Lee, W. Bang, C. S.Lee. Inter. J. Fatigue. 32, 1835 (2010)

5. Y. Gao, Q. D. Wang, J. H. Gu, Y. Zhao, Y. Tong. Mater. Sci. Eng. A. 459, 117(2007)

6. L. Wu, A.Jain, D.W. Brown, G.M. Stonica, S.R. Agnew, B. Clausen, D.E. Fielden, P.K Liaw. Acta Mater. 56, 688(2008)

7. L.J. Chen, C.Y. Wang, W. Wu, G.M. Stonica, L. Wu, P.K. Liaw. Metall. Mater. Trans. A. 38, 2235(2007) 